\title{
PSMC2 knockdown suppressed tumor progression of skin
} cutaneous melanoma

\author{
Yanwen Yang ${ }^{1}$, Fazhi Qi $\mathbb{D}^{1 凶}{ }^{凶}$, Chuanyuan Wei ${ }^{1}$, Jiaqi Liu $^{1}$, Yong Zhang ${ }^{1}$, Wenjie Luan ${ }^{1}$ and Jianying Gu ${ }^{1}$ \\ (c) The Author(s) 2021
}

Skin cutaneous melanoma (SKCM) is the most lethal tumor among three of the major malignant cancers of the skin. The mechanism underlying the malignant biological behaviors of SKCM is not fully clear. Our study intended to verify the molecular mechanism of proteasome $26 \mathrm{~S}$ subunit ATPase 2 (PSMC2) in malignant biological behaviors of SKCM. The Cancer Genome Atlas (TCGA) database was used to analyze the expression of PSMC2 in SKCM and its impact on prognosis. PSMC2 expression in 105 paired SKCM tissues was investigated by immunohistochemistry (IHC), its functional roles were verified using a series of cell experiments, and the underlying pathway was detected by protein-chip technology and gene set enrichment analysis. We found that PSMC2 was significantly upregulated in SKCN patients from TCGA datasets and verified in clinical SKCM tissues. Moreover, high PSMC2 was shown to closely correlate with the pathological stages and lymphatic metastasis of SKCM patients. Functionally, knockdown of PSMC2 suppressed the progression of SKCM through inhibiting cell proliferation, migration, and DNA damage in vitro as well as cell growth in vivo, whereas inducing apoptosis, cycle arrest in G2 phase. Similarly, pharmaceutical inhibition of proteasome with MG132 mimicked the PSMC2 knockdown induced defects in cell cycle arrest, apoptosis and proliferation, while overexpression of PSMC2 has the opposite effects. Mechanistically, the silence of PSMC2 remarkably elevated the pro-apoptotic proteins DR6, IGFBP-4, p21, and p53, while inhibited the anti-apoptosis protein TRAILR-3 and the proteins related to the Wnt signaling pathway. The present study revealed that PSMC2 participated in a positive regulation to promote the progression of SKCM through regulating the Wnt signaling pathway. Our findings may offer a new mechanism underlying the development and progression of SKCM, and a deeper understanding of PSMC2 may contribute to SKCM treatment.

Cell Death Discovery (2021)7:323; https://doi.org/10.1038/s41420-021-00727-2

\section{HIGHLIGHTS}

1. PSMC2 was found to be significantly upregulated in SKCM patients.

2. High PSMC2 expression was shown to closely correlate with the pathological stages and lymphatic metastasis of SKCM patients.

3. Knockdown of PSMC2 suppressed the progression of SKCM through inhibiting cell proliferation, migration, and DNA damage in vitro as well as cell growth in vivo, whereas inducing apoptosis, cycle arrest in the G2 phase.

4. Mechanistically, the silence of PSMC2 remarkably elevated the pro-apoptotic proteins DR6, IGFBP-4, p21, and p53, while inhibited the anti-apoptosis protein TRAILR-3 and the proteins related to the Wnt signaling pathway.

\section{INTRODUCTION}

Cutaneous melanoma (CM) is a common skin malignant tumor with a high fatality rate, degree of malignancy, rapid progress, strong invasiveness, and poor prognosis [1]. Epidemiological studies show that the incidence of SKCM is gradually increasing with more than 20,000 new cases of SKCM every year and is one of the more rapidly improving incidence rates among malignant tumors in China [2]. Surgery is the primary and standard treatment for SKCM [3]. After surgical treatment, the 5-year survival rate of SKCM is as high as $90 \%$ in the early stage $(\mathrm{I}, \mathrm{II})$ when there are no specific clinical manifestations, but unfortunately, most SKCM patients diagnosed is an advanced stage when the 5-year survival rate is less than $10 \%[4,5]$. There is no standard therapy for advanced-stage SKCM. Although great progress was recorded in both the understanding of SKCM biology and genetics and biological immunization therapies of early diagnosis and prevention, this malignancy became a burden on health care worldwide due to its increasing incidence and the lack of effective advanced treatment [6]. Usually, many clinical features of patients with SKCM in China differ from SKCM patients in the West [3]. Therefore, it is essential to explore the pathogenesis and seek new therapy for patients with SKCM.

Abnormal expression of biomarker proteins can be used as the basis for early diagnosis of SKCM. Growing evidence showed that proteasome $26 \mathrm{~S}$ subunit ATPase 2 (PSMC2) was able to promote tumor progression as a functional gene among several types of human tumors, including ovarian cancer [7], colorectal cancer (CC) [8], osteosarcoma [9], pancreatic cancer [10], and hepatocellular carcinoma [11]. PSMC2 is a vital member of the $19 \mathrm{~S}$ regulatory

${ }^{1}$ Department of Plastic Surgery, Zhongshan Hospital Fudan University, Shanghai 200065, China. ${ }^{凶}$ email: qifazhi002@163.com 
A

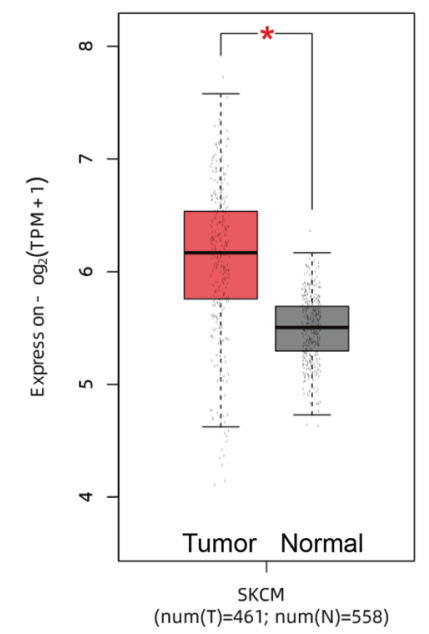

B

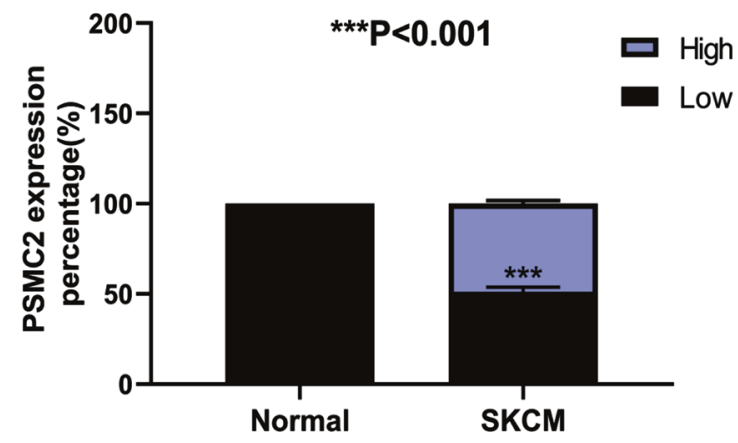

C

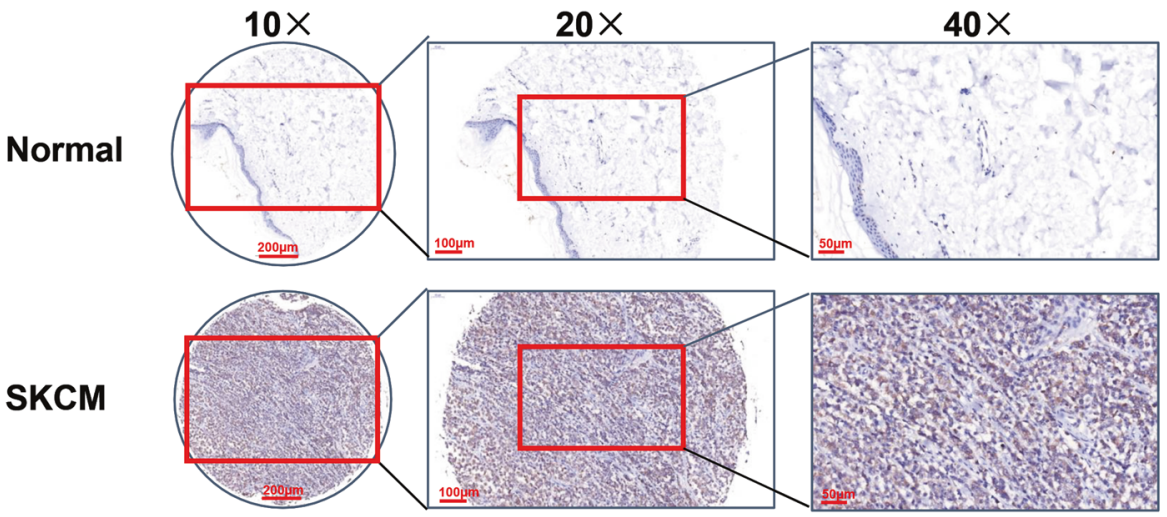

Fig. 1 PSMC2 expression in SKCM. A The expression of PSMC2 was detected from the TCGA dataset by using GEPIA2 (http://gepia.cancerpku.cn/). Red represents tumor samples and gray means normal samples. B Immunohistochemistry (IHC) revealed higher expression patterns of PSMC2 in SKCM tissues than that in normal skin tissues. $N=105$. C Representative images of IHC staining for PSMC2 expression in SKCM and normal tissues. Data were expressed as the mean \pm SD. ${ }^{*} P<0.05$ and ${ }^{* * *} P<0.001$.

subunit of the $26 \mathrm{~S}$ proteasome located on 7q22.1-q22.3 in the genome; it participated in regulating cell differentiation and proliferation, apoptosis, DNA damage repair, energy metabolism, and signal transduction [12]. PSMC2 suppression decreased cell proliferation in ovarian cancer and was also correlated with pancreatic cancer cell proliferation and apoptosis [7, 10]. PSMC2 was expressed highly in osteosarcoma patients; knockdown of PSMC2 reduced cell proliferation, migration, and increased apoptosis, which indicated that PSMC2 act as an oncogene for osteosarcoma [9]. Whether PSMC2 is involved in the occurrence and development of SKCM has not been investigated.

Here in this study, we first proved the abnormal expression of PSMC2 in SKCM tissue using gene-chip technology, then explored the biological significance and clinical relevance of PSMC2 with SKCM patients, and exam the underlying molecular mechanism of PSMC2 in SKCM last.

\section{RESULTS}

A high level of PSMC2 was closely associated with clinical characteristics of patients with SKCM

To determine the expression of PSMC2 in SKCM, we first analyzed the mRNA expression profiles and the associated clinical characteristics in 461 cases of SKCM samples compared to 558 normal samples from The Cancer Genome Atlas (TCGA) dataset. A striking upregulation of PSMC2 were observed in SKCM samples (Fig. 1A, $P<0.05$ ) from TCGA, which might imply an oncogenic role of PSMC2 in the development of SKCM. Next, we determined the expression level of PSMC2 in the SKCM clinical samples, which were collected from our hospital. As shown in Fig. 1B, C, the expression of PSMC2 was significantly upregulated in human SKCM samples compared with the normal para-cancer skin tissues. Clinically, we were pleasantly surprised that SKCM patients with high PSMC2 expression were positively related to the pathological stages and lymphatic metastasis $(\mathrm{N})$ (Table 1). These results suggested that PSMC2 was increased with the deepening of the malignancy degree of SKCM and might serve as a potential prognostic marker for patients with SKCM.

Knockdown of PSMC2 suppressed cell proliferation, migration, apoptosis, and DNA damage in SKCM cells, as well as cell growth in vivo

Firstly, higher PSMC2 levels were observed in three human SKCM cell lines (A375, SK-MEL-1, and SK-MEL-28) comparing to the human normal epidermal melanocytes (PIG1) (Fig. 2A). After showing the overexpression of PSMC2 in SKCM cells, we then investigated the potential biological roles of PSMC2 in SKCM through a series of loss-of-function assays. Due to A375 cells owning relatively high PSMC2 levels, three lentivirus-mediated shRNAs targeting PSMC2 (shPSMC2-1, shPSMC2-2, and shPSMC2-3) were constructed to knockdown PSMC2 in A375 cells. After validating transfection efficiency through detecting the PSMC2 expression and cell proliferation (Fig. 2B, C), PSMC2 shRNA3 was selected for the subsequent experiments and then 
Table 1. Relationship between PSMC2 expression and clinical characteristics in patients with skin cutaneous melanoma.

\begin{tabular}{|c|c|c|c|c|}
\hline Features & $\begin{array}{l}\text { No. } \\
\text { of cases }\end{array}$ & $\begin{array}{l}\text { PSMC2 } \\
\text { expression } \\
\text { low }\end{array}$ & high & $p$ value \\
\hline All cases & 105 & 60 & 45 & \\
\hline Age (years) & & & & 0.104 \\
\hline$\leq 54$ & 54 & 35 & 19 & \\
\hline å 54 & 51 & 25 & 26 & \\
\hline Gender & & & & 0.611 \\
\hline Male & 59 & 35 & 24 & \\
\hline Female & 46 & 25 & 21 & \\
\hline T Infiltrate & & & & 0.074 \\
\hline $\mathrm{T} 2$ & 6 & 3 & 3 & \\
\hline T3 & 28 & 12 & 16 & \\
\hline T4 & 71 & 45 & 26 & \\
\hline $\begin{array}{l}\text { lymphatic } \\
\text { metastasis (N) }\end{array}$ & & & & $0.000^{* * * *}$ \\
\hline NO & 91 & 60 & 31 & \\
\hline N1 & 10 & 0 & 10 & \\
\hline $\mathrm{N} 2$ & 4 & 0 & 4 & \\
\hline Stage & & & & $0.000^{* * * *}$ \\
\hline 1 & 6 & 3 & 3 & \\
\hline II & 83 & 57 & 26 & \\
\hline III & 12 & 0 & 12 & \\
\hline IV & 4 & 0 & 4 & \\
\hline
\end{tabular}

collectively called shPSMC2. Subsequently, shPSMC2 effectively suppressed the expression of protein and mRNA levels both in A375 (Fig. 2D, E) and SK-MEL-28 cells (Fig. 2F, G). As predicted, knockdown of PSMC2 both in A375 and SK-MEL-28 cells was ready to suppress cell proliferation by CCK-8 assay (Fig. 3A, B). The lessened cell growth could be attributed to cell cycle distribution disorder and increased apoptosis [13]. Flow cytometry was performed to further analyze cell apoptosis and cell cycle distribution in SKCM cells to verify the above point. Furthermore, we found that PSMC2 knockdown elicited a remarkable increase of apoptotic population of A375 and SK-MEL-28 cells (Fig. 3C, D). Besides, the depletion of PSMC2 in A375 cells leads to a significant arrest in the G2 phase (Fig. 3E). Similarly, an increasing cells population in the G2 phase was also observed in SK-MEL-28 cells transfected with shPSMC2 (Fig. 3F). Next, we found that PSMC2 knockdown remarkably inhibited cell migration of A375 and SKMEL-28 cells through wound healing and Transwell assays (Fig. $4 A-D)$. Due to $y-H 2 A X, A T M$ and CDKN1A were the key proteins response to DNA damage, we found that PSMC2 depletion could remarkably inhibit the protein expression of $\mathrm{Y}-\mathrm{H} 2 \mathrm{AX}, \mathrm{ATM}$, and CDKN1A both in A375 and SK-MEL-28 cell lines (Fig. 4E). Meanwhile, the results of the tumor-forming experiment in nude mice showed that the tumor volume and weight in the shPSMC2 group were significantly lower than those in the control group (Figs. 4F, G). These results strongly support that the silencing of PSMC2 contributes to inhibiting malignant phenotypes and tumor progression of SKCM in vitro and in vivo.

\section{Inhibiting PSMC2 induces SKCM cell growth suppression, increased cell cycle arrest, and enhances apoptosis, while overexpression of PSMC2 promote SKCM growth}

As PSMC2 is a crucial element for proteasome assembly and functional performance, we deduced PSMC2 depletion causing biological abnormalities in SKCM cells might associate with disabled proteasome activity. To explore this surmise, MG132, a selective inhibitor of the proteasome, was added to A375 and SKMEL-28 SKCM cells to down-regulate PSMC2 expression (Fig. $5 A-D)$. Meanwhile, lentivirus transduction (OE-PSMC2) was used to specifically upregulate PSMC2 expression in two SKCM cells (Fig. 5A-D). Expectedly, MG132 was able to suppress cell growth (Fig. $5 \mathrm{E}, \mathrm{F}$ ), while to elevate the cell population in the G2/M phase (Fig. $5 \mathrm{G}, \mathrm{H}$ ), and promoting cell apoptosis (Fig. 5I, J). In addition, overexpression of PSMC2 remarkably promoted cell growth of A375 and SK-MEL-28 cells via CCK-8 assay (Fig. 5E, F). Inconsistent, cell population in the $\mathrm{G} 2 / \mathrm{M}$ phase was obviously suppressed in PSMC2 overexpressing cells (Fig. 5G, H), as well as cell apoptosis was restrained (Fig. 5l, J). Herein, we proved proteasome is necessary for SKCM growth due to its inhibition or deficiency for PSMC2 would lead to the biological function defects of SKCM cells. Additionally, these findings also indicated that exogenous enhanced PSMC2 expression could apply a valid effect on SKCM growth.

Silencing of PSMC2 alters apoptosis-related gene expression and enrichment of related pathways in SKCM cells

To further elucidate the underlying molecular mechanism of PSMC2 in direct SKCM progression, a protein chip assay was conducted to discover genes affected by PSMC2 silencing. As p21 was one of the most strongly upregulated genes and p53 was upregulated at the same time, we mainly focused on the role of the p53/p21 axis in this study. As shown in Fig. 6A, B, we found that the differential expression of 43 human apoptotic-related proteins in SK-MEL-28 cells after PSMC2 knockdown was analyzed using a human apoptosis antibody array. Moreover, the proapoptotic proteins were overtly increased includes DR6, IGFBP-4, p21, and p53, while anti-apoptosis protein TRAILR-3 was obviously decreased in the shPSMC2 group comparing to the shCtrl group (Fig. 6C). Notably, p21 and p53 were most strongly upregulated among these pro-apoptotic proteins. Collectively, these results indicated that PSMC2 was likely involved in apoptosis-related signaling pathways, especially the p53/p21 signaling pathway, in SKCM progression. These results match the previous cellular experiments, especially the cell apoptosis assay.

To confirm the downstream associated molecular mechanisms underlying PSMC2 in SKCM cells, the enrichment analysis of related-pathway was performed using GSEA. The GSEA enrichment snapshot showed that most genes involved in the Wnt signaling pathway were found in the region related to PSMC2 in SKCM cells (Fig. 6D). To further verify the GSEA results, we performed western blotting and discovered the proteins expression levels of GSK-3 $\beta$ and $\beta$-catenin, key genes of the Wnt signaling pathway, were all significantly decreased in PSMC2 knockdown cells in comparison with control cells (Fig. 6E). Combining these bioinformatics results, we speculated that the Wnt signaling pathway could influence the functions of PSMC2, thereby participating in SKCM progression.

\section{DISCUSSION}

SKCM is a complex disorder characterized by high heterogeneity that places it among the most aggressive type of cancer [4], however related research about functional verification and mechanism of SKCM2 in human cancers has still been lacking. In the present study, we aimed to verify the function of PSMC2 on the malignant biological behaviors of SKCM. By conducting gene microarray analysis in the TCGA database and using $I H C$, we evaluated the upregulation of PSMC2 in SKCM tissues and found that high PSMC2 expression was closely correlated with the pathological stages and lymphatic metastasis of SKCM patients. Then, we demonstrated that PSMC2 expression was necessary for SKCM cell malignant progressions by reporting that PSMC2 was over-expressed in SKCM cell lines, which was necessary for cell 
A

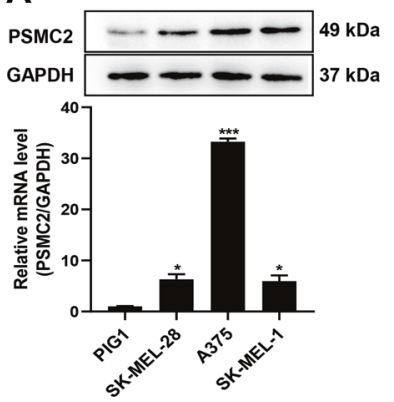

D
B

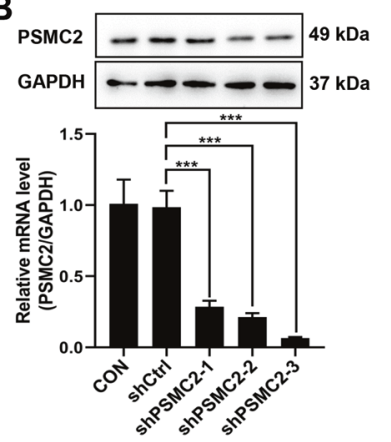

E
C

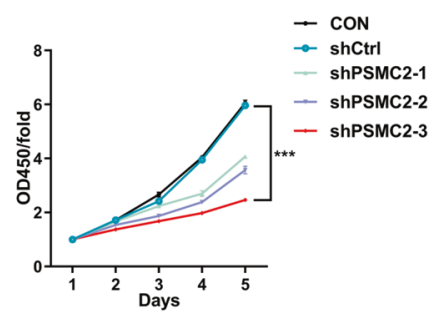

A375
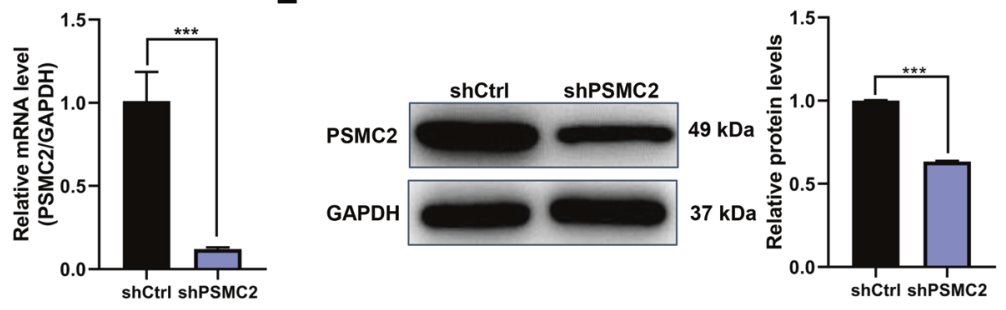

F

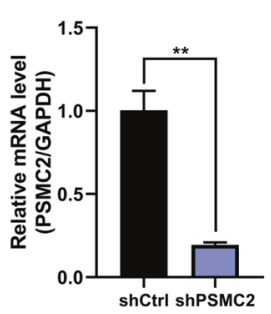

G
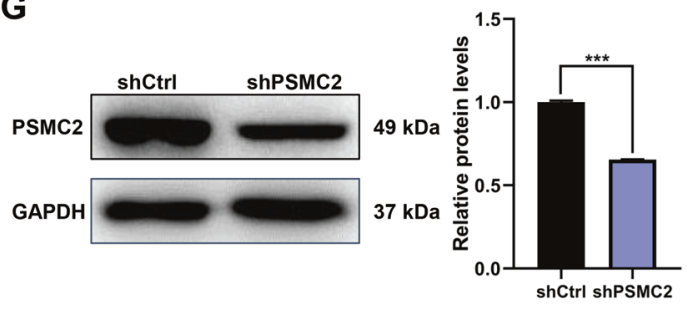

Fig. 2 PSMC2 shRNAs transfections. A The PSMC2 expression profile at the protein level (upper part) and mRNA level (bottom part) in human melanoma cell lines (A375, SK-MEL-1, SK-MEL-28) and human epidermal melanocytes (PIG1). B Western blot (upper part) and RT-qPCR (bottom part) were used to assess the transfection efficiency of PSMC2 shRNA-1 and shRNA-2 and shRNA-3 in A375 cells. C PSMC2 knockdown is associated with reduced proliferation in A375. CCK-8 assay was performed to measure the rate of cell proliferation of A375 after transfected with shPSMC2-1, shPSMC2-2, and shPSMC2-3 for 5 days. D, E RT-qPCR and Western blot were used to detect the expression of PSMC2 in A375 cells after shPSMC2 transfection. F, G RT-qPCR and Western blot were used to detect the expression of PSMC2 in SK-MEL-28 cells after shPSMC2 transfection. Each independent experiment was repeated at least three times. Data were expressed as the mean \pm SD. ${ }^{*} p<0.05$, ${ }^{* *} p<0.01$ and ${ }^{* * *} p<0.001$.

proliferation, migration, apoptosis, and cell cycle regulation. Further GSEA pathway enrichment and western blot analysis revealed that the function of PMSC2 was closely related to the Wnt signaling pathway in SKCM progression. Taken together, we provided compelling evidence for the role of PSMC2 in tumorigenesis and progression of SKCM via regulating the Wnt signaling pathway, supporting that PSMC2 may be an oncogenic gene participating in SKCM progressions.

High PSMC2 expression was closely correlated with the pathological stages and lymphatic metastasis of SKCM patients. A survival analysis showed that the OS of high expression PSMC2 in colorectal cancer samples was lower than that with low expression PSMC2 [8], and patients with pancreatic cancer who expressed high PSMC2 had a poor prognosis [10]. Similarly, in our study, PSMC2 was closely associated with the pathological stages and lymphatic metastasis (N) of SKCM patients, and that PSMC2 expression was ubiquitously expressed in SKCM samples than in the adjacent para-cancer skin tissues, and overexpressed SKCM cell lines, as A375, SK-MEL-1, and SK-MEL-28 than PIG1 cell line. This suggests that PSMC2 may work as a biomarker protein, and can be used as the basis for early diagnosis of SKCM.

Here we suggest that PSMC2 knockdown remarkably inhibited SKCM cell DNA damage, caused SKCM cells cycle arrest in the G2 phase, and upregulated p53 and p21 expression by three evidence. First, besides inhibited cell proliferation, migration in vitro and inhibited tumor growth in vivo, we found that PSMC2 knockdown remarkably inhibited the DNA damage of SKCM cells. Among the expression decreased DNA damage-related proteins, as ATM, $\mathrm{Y}-\mathrm{H} 2 \mathrm{AX}$, and CDKN1A after PSMC2 knockdown in this study, CDKN1A was reported to play a critical role in DNA replication and cell-cycle progression at the G1/S checkpoint [14]. Second, the deficiency of PSMC2 also induced apoptosis and cell cycle arrest in the G2 phase. In our study, cell apoptosis induced by PSMC2 knockdown is complex and involved in multi-apoptotic proteins. He et al. found that silencing of PSMC2 significantly suppressed cell proliferation, enhanced apoptosis, and accelerated G2 phase and/or S phase arrest in CC cells [8]. Dissimilarly, our findings revealed that PSMC2 knockdown leads to apoptosis acceleration and cell cycle arrest only in the G2 phase of SKCM cells. Third, among the expression upregulated human apoptoticrelated markers, as DR6, IGFBP-4, p21, p53, and TRAILR-3 after PSMC2 knockdown, p21, a CDK inhibitor, was the most significant upregulated, and was reported to participate in cell cycle progression and could play a crucial role in tumor development by the p53 pathway [15-18]. Also, a previous study held that increased expression of PSMC2 was determined in tumor tissues from the p21-HBx transgenic mice [11]. After DNA damage, many cells appear to enter a sustained arrest in the G2 phase of the cell cycle [19]. Escape from apoptosis is an inducible factor for the occurrence of cancer [12]. Additionally, the G2/M checkpoint and DNA repair mechanisms deal with oxidative stress-induced DNA damage, and G2 arrest induced by oxidative stress suppressed 
A

A375
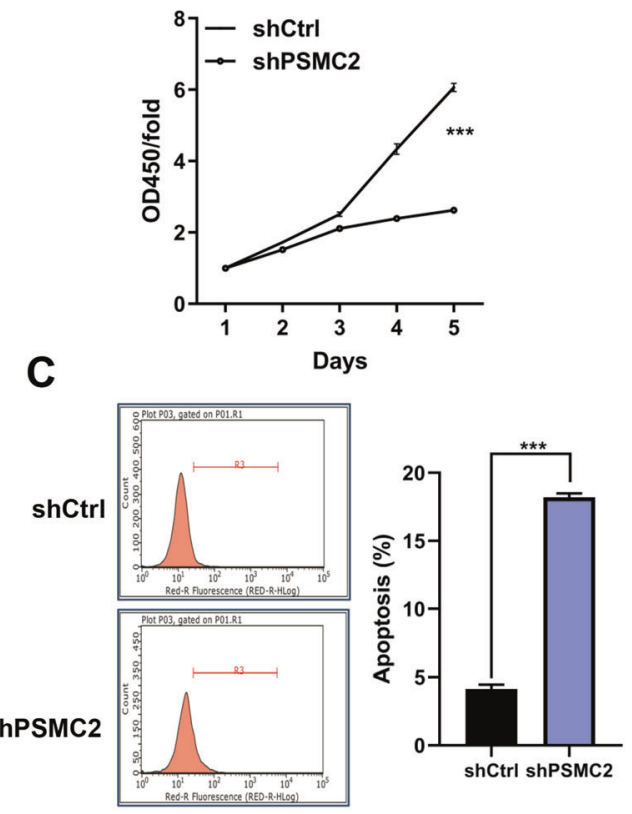

E

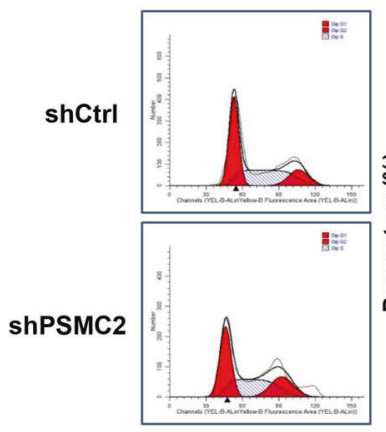

B

D

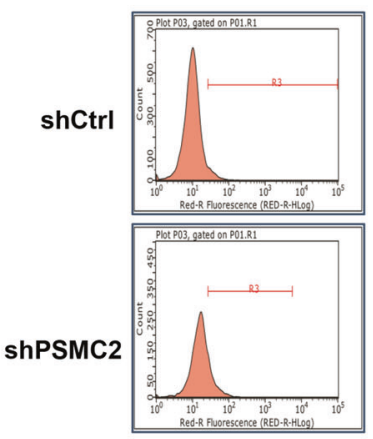

SK-MEL-28

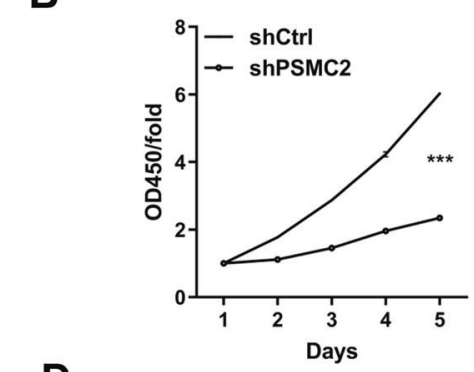$$
\text { . }
$$ 
A375
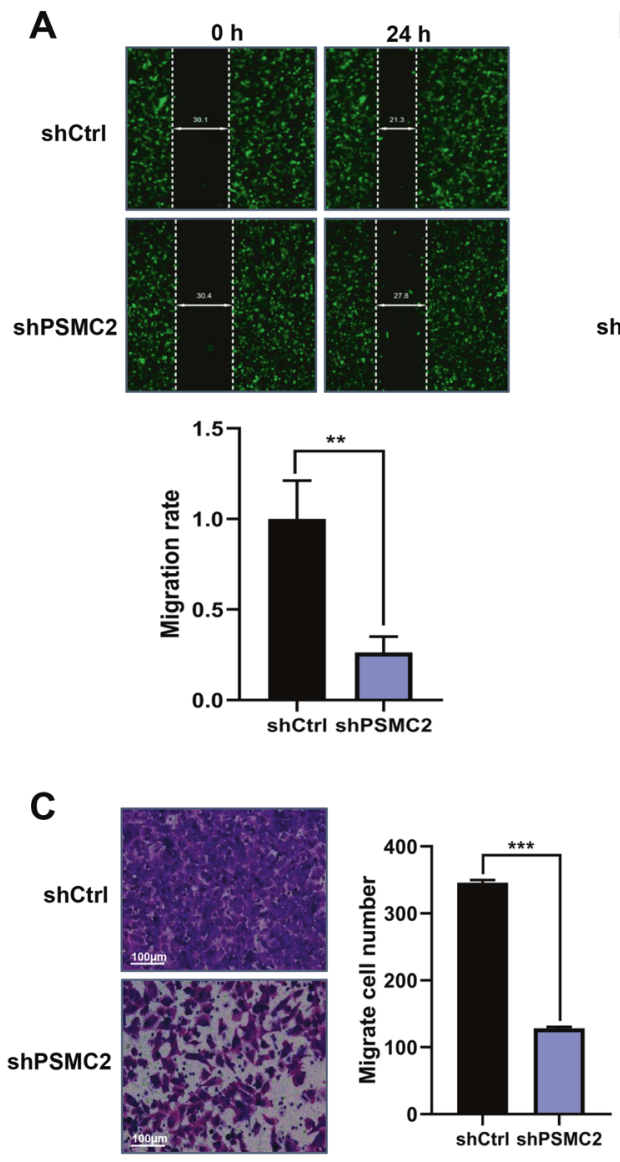
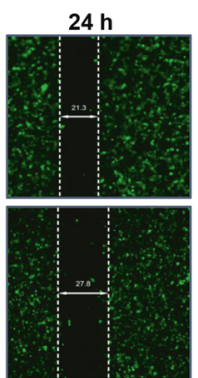

shCtrl shPSMC2
B

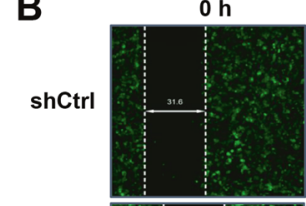

SK-MEL-28

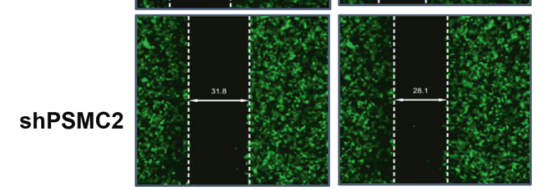

D
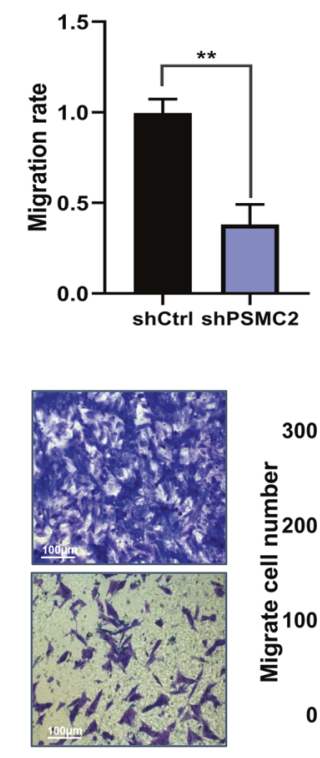

$8 \mathrm{~h}$
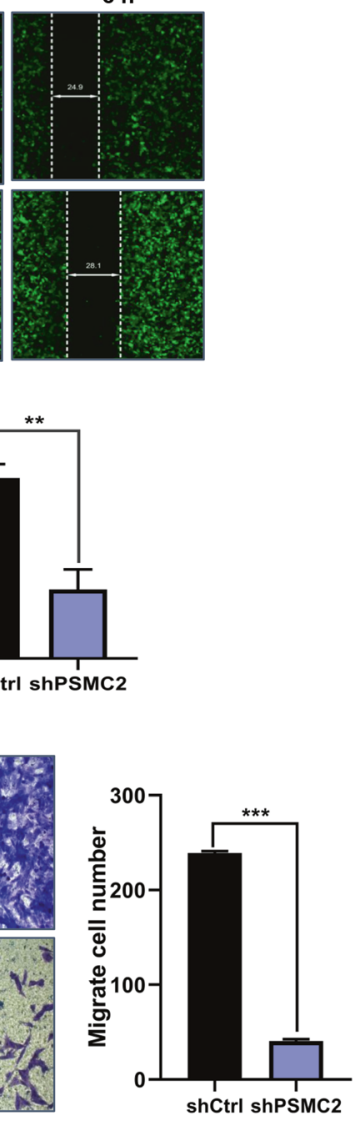
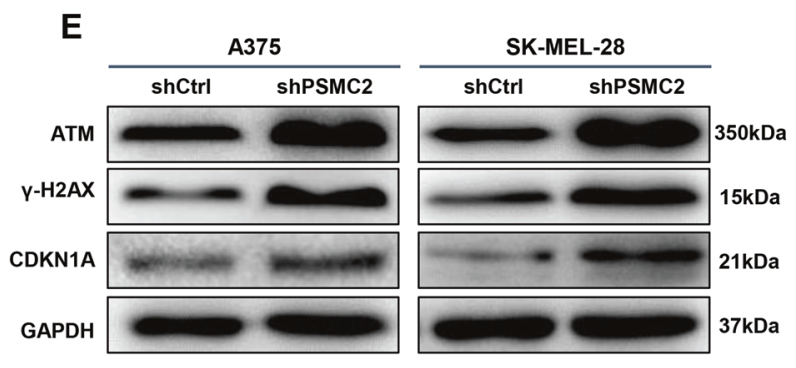

$\mathbf{F}$

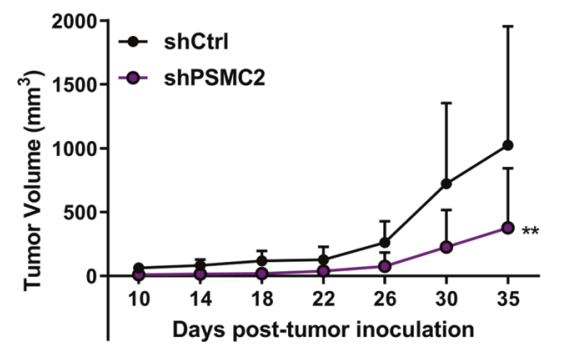

G
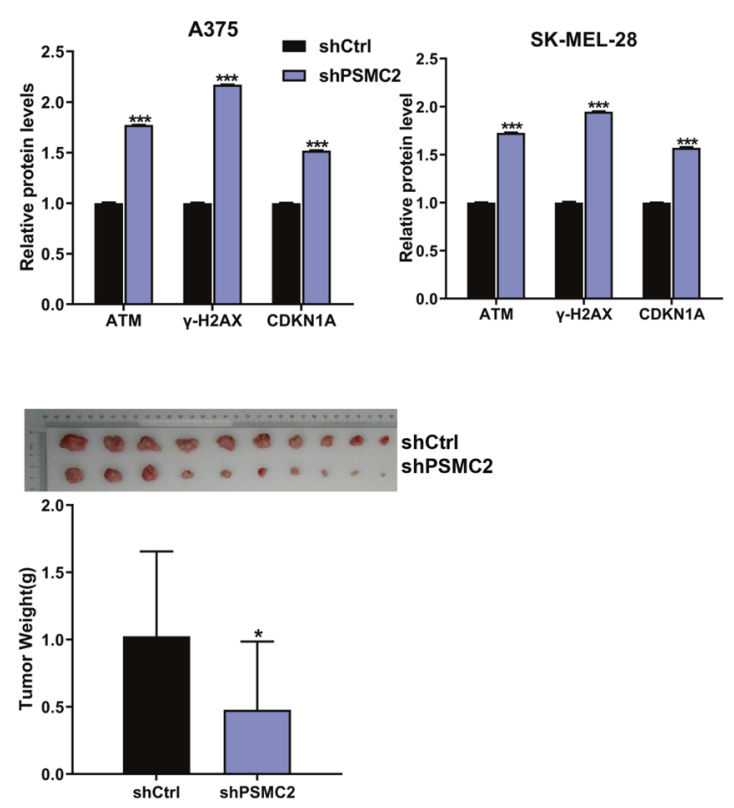

Patients and tissue samples

biological information analysis using The Cancer Genome Atlas (TCGA) database. The Mann-Whitney $U$ analysis was performed to assess the relationship between PSMC2 and the clinical characteristics of patients with SKCM.

One hundred five cases of SKCM tissues and adjacent normal tissues (ANT) were obtained from patients hospitalized in the Zhongshan Hospital, Fudan University, and no patients received radiotherapy or 
Fig. 4 Knockdown of PSMC2 inhibits cell migration and DNA damage in SKCM cells and tumor growth in vivo. A, B Scratch test assays were performed to detect cell migrative capacities of A375 (at 0 and $24 \mathrm{~h}$ ) and SK-MEL-28 cells (at 0 and $8 \mathrm{~h}$ ) after shPSMC2 transfection. C, D Transwell assays were performed to detect cell migrative capacities of A375 and SK-MEL-28 cells after shPSMC2 transfection. E Western blotting was used to evaluate the protein expressions of ATM, $\gamma-\mathrm{H} 2 \mathrm{AX}$, and CDKN1A in PSMC2 silenced A375 and SK-MEL-28 cells. GAPDH was used as a loading control. Histogram representing indicated the results of three independent experiments. $\mathbf{F}$ Tumor volume growth curves for subcutaneous xenografts and data were obtained from 10 animals in each group. G After scarification, xenograft excised tumors were recorded and the bar graph presented the excised tumor weights derived from control or PSMC2 silenced A375 cells. $n=10$. Each independent experiment was repeated at least three times. Data were expressed as the mean \pm SD. The statistical significance of the data between two groups was compared using Student's $t$-test. ${ }^{*} p<0.05,{ }^{* *} p<0.01$ and ${ }^{* * *} p<0.001$.

chemotherapy before surgical excision. Two pathologists independently diagnosed the histopathological features of SKCM tissues. This study was approved by the Ethics committee of the Research Department of Zhongshan Hospital Biomedical and conformed to the ethical principles set forth by the Declaration of Helsinki. Written informed consents were signed and obtained from all participants.

\section{Immunohistochemistry (IHC) staining}

SKCM tissues and ANT tissues were frozen and cut into 4-8 $\mu \mathrm{m}$ sections at room temperature for $30 \mathrm{~min}$. IHC staining is performed as described previously [26]. Briefly, sections were incubated with primary antibody against PSMC2 overnight at $4{ }^{\circ} \mathrm{C}$. Subsequently, sections were incubated with a specific secondary antibody for one $h$ at room temperature, followed by incubation with horseradish peroxidase (HRP)-labeled streptavidin for one $h$, then staining with diaminobenzidine (DAB) for $5 \mathrm{~min}$ and counterstaining with hematoxylin (Gene Tech, China). The results of IHC staining were assessed blindly by two experienced pathologists. Five randomly visual fields in each section were selected and then assessed by a light microscope to detect PSMC2 positive expression in SKCM tissues with a color of brownish-yellow regarded as positive. Average positive expression rate $=$ positive cells/total cells $\%$.

\section{Cell culture}

Three human SKCM cell lines (A375, SK-MEL-1, and SK-MEL-28), and human normal epidermal melanocytes (PIG1) were all purchased from the American Type Culture Collection (ATCC). A375 and SK-MEL-1 cells were cultured in Dulbecco's modified Eagle's medium (DMEM; Gibco, USA) with $20 \%$ FBS. SK-MEL-28 cells were cultured in MEM with $10 \%$ FBS. PIG1 cells were grown in the medium 254 (Cascade Biologics, USA). All cells were maintained in an atmosphere with $5 \% \mathrm{CO}_{2}$ at $37^{\circ} \mathrm{C}$. All cell lines used in this study passed STR identification by the purchase agency.

\section{Lentiviral shPSMC2 construction}

Small hairpin RNAs (shRNAs) was designed to target the human PSMC2 gene (Gene ID, 5701) according to its sequence in the NCBI database as following:

shPSMC2-1, 5'-GAGCTCACTGGTATTAAAGAA-3';

shPSMC2-2, 5'-CAACGTAAAGCAGTTGGCAA-3 ';

shPSMC2-3, 5 '-AAGCAAGTTGAAGATGACATT-3'.

Lentivirus without shRNA insert was used as a control. The fragments of shRNA were inserted into the lentivirus vector and transfected into 293 T cells with packaging vectors using Lipofectamine 3000 (Invitrogen) as soon as cell density reached $70 \%$. The recombinant lentivirus (OE-PSMC2) containing PSMC2 were purchased from GeneChem (Shanghai, China). After lentivirus construction, cells were transfected with lentivirus for establishing the over-expressing PSMC2 SKCM cells. After $48 \mathrm{~h}$ of transfection, the recombinant lentivirus was collected from a cell culture medium for further infection. Lentivirus was concentrated using the centrifugal ultrafiltration device (Millipore), and then stored at $-80^{\circ} \mathrm{C}$.

\section{CCK-8 assay}

CCK-8 assay was performed to assess the cell proliferation of SKCM cells. Briefly, $48 \mathrm{~h}$ after infection, cells were collected and plated into 96-well plates $\left(2 \times 10^{3}\right.$ cells/per well). CCK-8 solution was added to each well (containing $200 \mu \mathrm{L}$ medium) and further cultured for $2 \mathrm{~h}$ at $37^{\circ} \mathrm{C}$. The absorbance at $450 \mathrm{~nm}$ was detected under an absorbance microplate reader. This absorbance is directly proportional to the number of living cells in the following five days.

\section{Wound Healing}

After infection for $48 \mathrm{~h}$, a total of $100 \mu \mathrm{L}$ cells were collected and seeded into 24-well plates with a density of $5 \times 10^{5}$ cells $/ \mathrm{ml}$, followed by routine culture to form a cell monolayer. Then, cells were scratched, washed, cultured with the medium containing $10 \mathrm{~g} / \mathrm{L}$ bovine serum albumin (BSA) and $1 \% \mathrm{FBS}$, and placed under a microscope for measuring the migration distance. Cells were then continuing to maintain in the medium containing $10 \%$ FBS. After $8-24 \mathrm{~h}$, the relative distance of cells migrating to the wounded area was observed again under a microscope.

\section{Transwell assay}

After being starved for $24 \mathrm{~h}$ in the serum-free medium, cells were washed with PBS twice and then resuspended with serum-free Opti-MEM (Invitrogen) with the cell density of $5 \times 10^{4} \mathrm{cell} / \mathrm{s} / \mathrm{ml}$. Then, cells were resuspended and seeded into the upper chambers of the 24-well Transwell plate $(8 \mu \mathrm{m}$, Corning Incorporated, Corning, NY), with triplet for each sample and $100 \mu \mathrm{L}$ in each well. Next, $600 \mu \mathrm{L}$ of DMEM or MEM culture medium was added into the lower chamber. After incubation for $48 \mathrm{~h}$, cells were fixed with $4 \%$ paraformaldehyde for $10 \mathrm{~min}$, washed with PBS three times, and stained with crystal violet for an additional $10 \mathrm{~min}$. After washing with PBS and dried, cell invasion was observed under a microscope, and five random sights were selected to count the cells that crossed the membrane.

\section{Apoptosis and cell cycle assay}

After lentivirus infection, A375 and SK-MEL-28 cells were assessed with a flow cytometer (BD Biosciences, San Diego, CA, USA) following the manufacturer's instruction. Briefly, cells were trypsinized, washed with PBS, and centrifugated at $1300 \mathrm{rpm}$ for $5 \mathrm{~min}$. Subsequently, cells were washed once in $1 \times$ binding buffer and resuspended in $1 \times$ staining buffer. $100 \mu \mathrm{L}$ cell $\left(5 \times 10^{5}\right.$ cells) were then incubated with $10 \mu \mathrm{L}$ Annexin V-APC apoptosis detection Kit II or $50 \mu \mathrm{g} / \mathrm{mL}$ PI mixed with $0.1 \mathrm{mg} / \mathrm{ml}$ RNaseA and $0.1 \%$ Triton X-100 at room temperature for 15 or $30 \mathrm{~min}$ in the dark. Cell apoptosis and the percentage of cells in a different phase (G1, S, and G2) were analyzed by Cell Quest software (BD Biosciences, USA).

\section{Xenotransplant murine models}

We carried out animal xenograft model studies by institutional guidelines; The right armpit region of 6-week female BALB/c nude mice was given the subcutaneous injection with $0.2 \mathrm{~mL}$ exponentially growing lentivirus infected A375 cells $\left(2 \times 10^{6} \mathrm{cell} / \mathrm{ml}\right)$. When the tumors reached an average of $100 \mathrm{~mm}^{3}$, the mice were randomly divided into two groups $(n=10$ mice in each group). On the 10th day after injection, we measured tumor diameters every four days by a caliper. Mice were euthanized on the 35th day after injection. Then the tumors were obtained and weighted following necropsy. Finally, the formula (length $\times$ width $^{2} \times 1 / 2 \mathrm{~mm} 3$ ) was used to calculate tumor volume at days $10,14,18,22,26,30$, and 35 post tumor inoculations. All animal procedures were approved by the Institutional Animal Care and Use Committee of the Research Department of Zhongshan Hospital Biomedical.

\section{Inhibition of proteasome in SKCM cells}

The most common agent to inhibit proteasome in experiments is MG132 (a selective proteasome inhibitor, Sigma-Aldrich). Subsequently, A375 and SK-MEL-28 SKCM cells treated with MG132 $(30 \mu \mathrm{m} / \mathrm{L})$ were regarded as proteasome-blocking cells.

\section{Protein chip assay}

After shPSMC2 transfection, the apoptosis signaling pathways underlying PSMC2 in SK-MEL-28 cells were detected by the Human apoptosis 
A

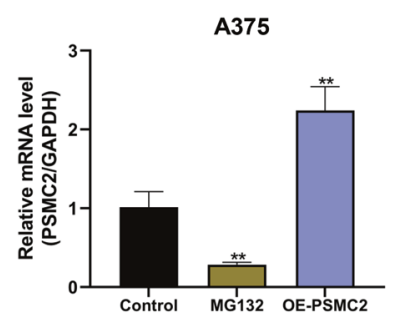

C

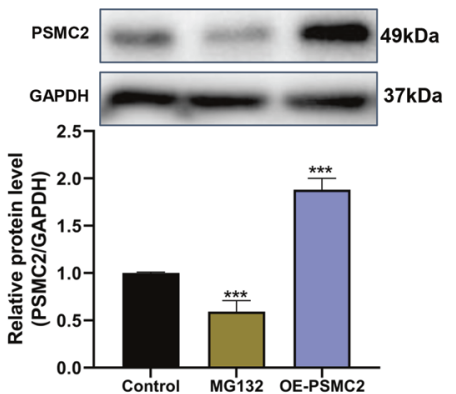

E

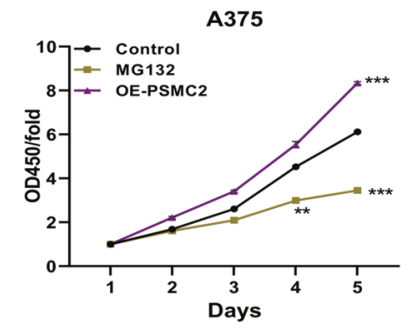

G
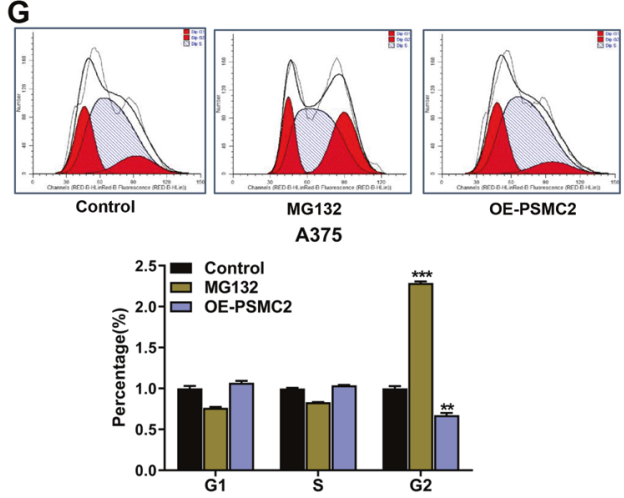

I
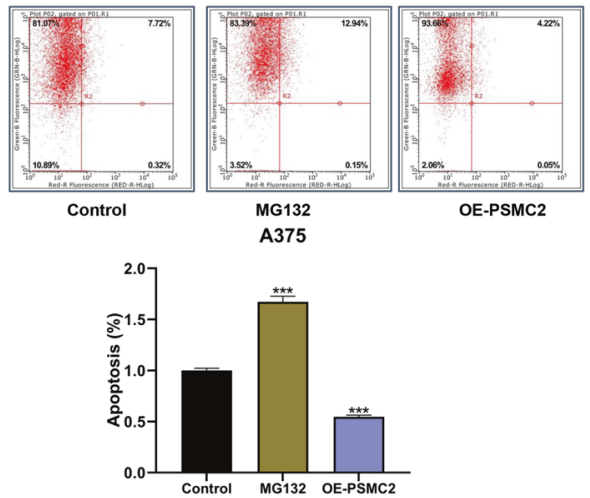

B

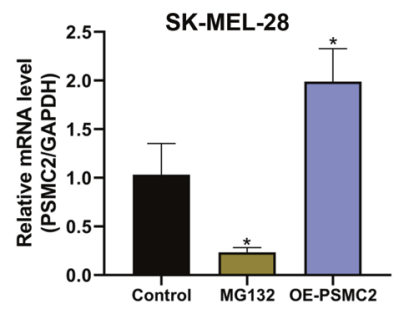

D

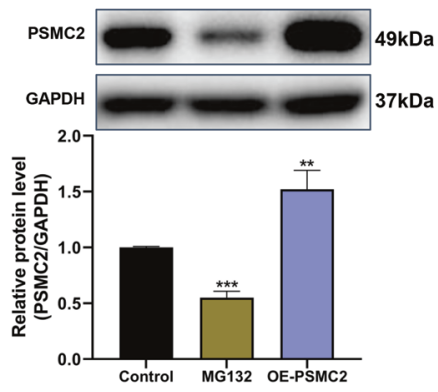

SK-MEL-28

F

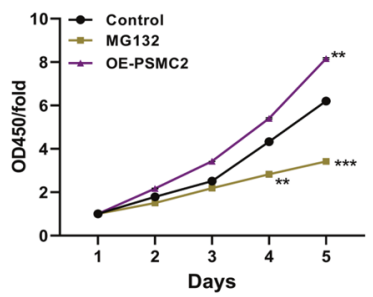

H
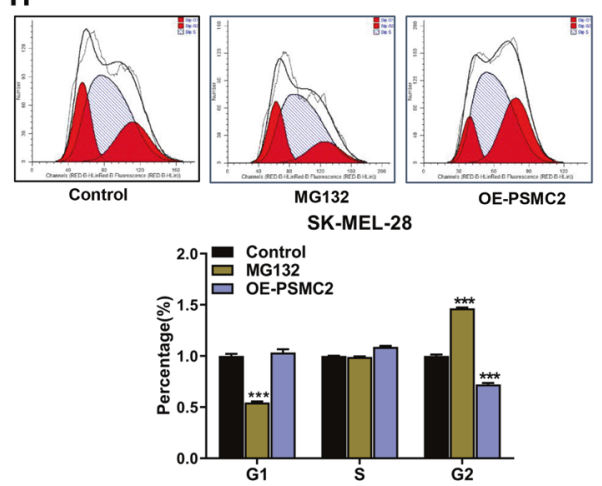

J

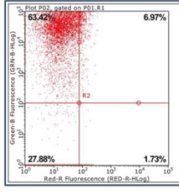

Control

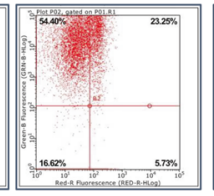

MG132

SK-MEL-28

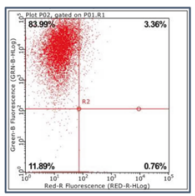

OE-PSMC2

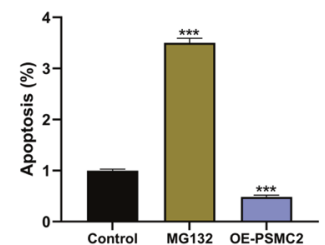

Fig. 5 The effect of inhibiting proteasome and exogenously enforced PSMC2 expression on cell proliferation, cell cycle and apoptosis. After treatment with MG132 or OE-PSMC2 plasmids, (A, B) The mRNA levels of PSMC2 were assessed by real-time PCR in A375 and SK-MEL-28 cells. C, D) The protein levels of PSMC2 were determined by western blot in A375 and SK-MEL-28 cells. E, F The consequent influences on SKCM cell proliferation of A375 and SK-MEL-28 cells were separately determined by the CCK-8 assay. G, H Flow cytometry analysis was used to evaluate the cell cycle progression and cell fractional quantification in the G0/G1, S, and G2/M phases were shown in the diagrams. I, J Apoptosis via quantifying Annexin FITC positive cells were shown and the apoptotic rate was calculated as the percentage of Annexin APC positive cells. Each independent experiment was repeated at least three times. Data were expressed as the mean \pm SD. ${ }^{*} p<0.05,{ }^{* *} p<0.01$ and $* * * p<0.001$. 
A

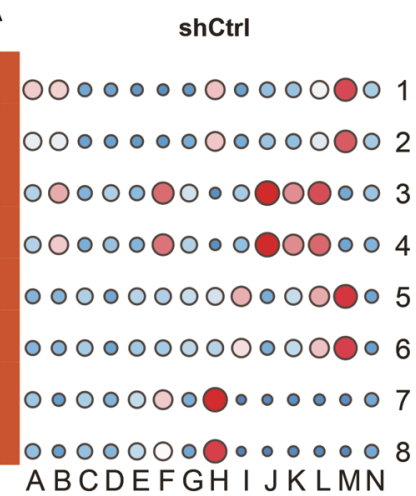

shPSMC2

$\begin{array}{ll}000000000000001 & \bullet 20 \\ 000000000000002 & 040 \\ 000000000000003 & 080 \\ 000000000000004 & 0100 \\ 000000000000005 & 120 \\ 0 & 140 \\ 00000000000006 & 160\end{array}$

000000000000007

000000000000008

A BCDEFGH I JKLMN

B

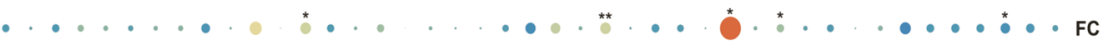

$\ldots \ldots \ldots \ldots \ldots \ldots \ldots$ shttrl

$\ldots \ldots \ldots . . . \ldots \ldots$ shPSMC2

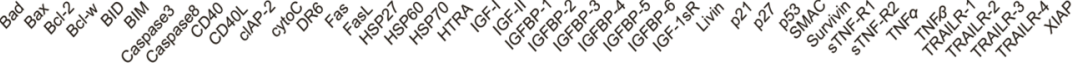

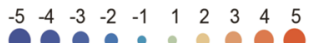

C
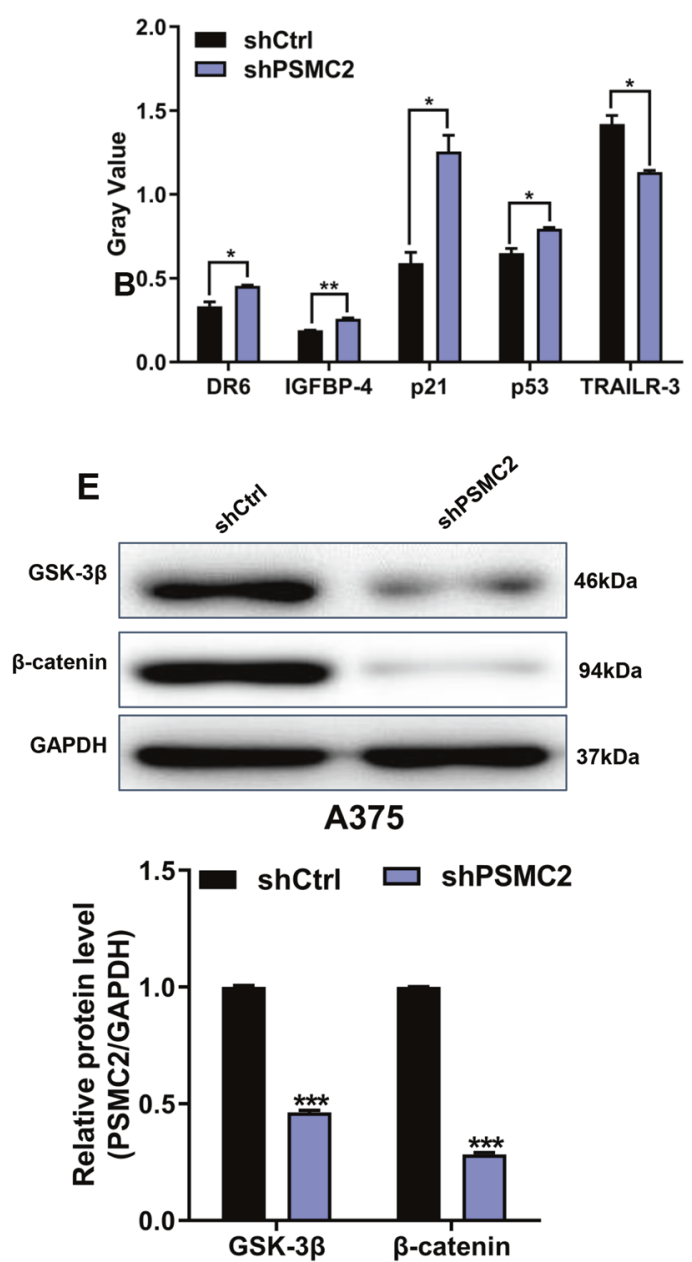

D
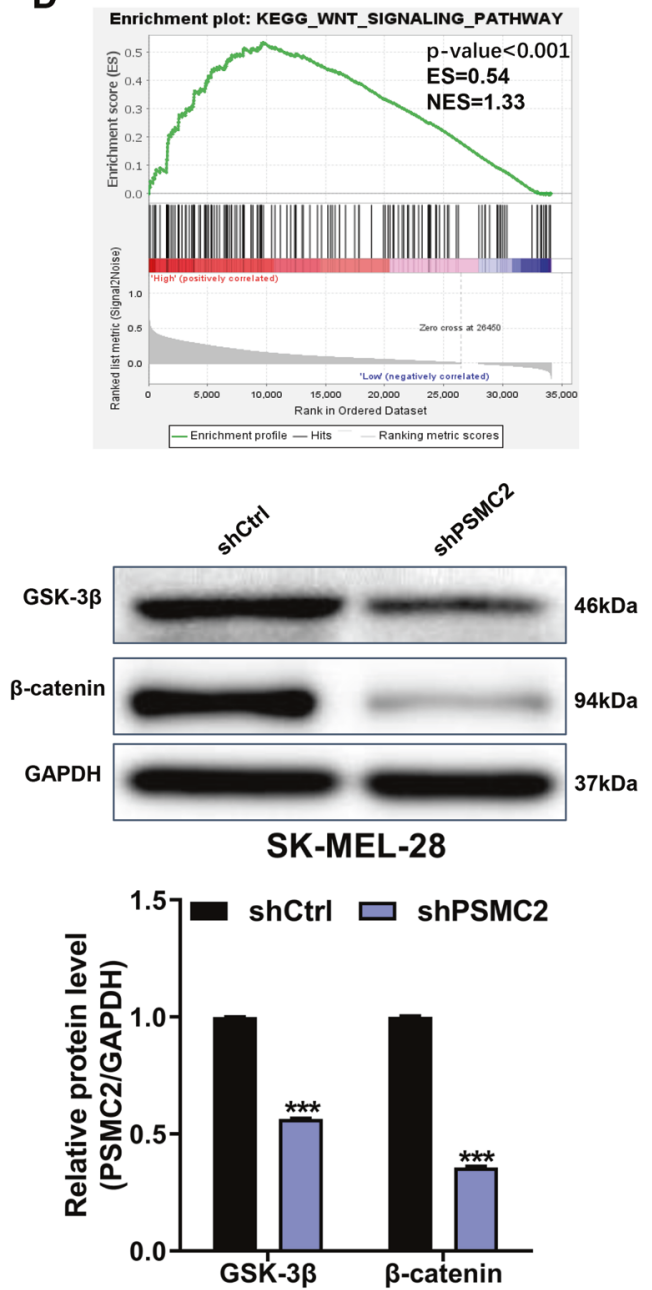

Fig. 6 Exploring the downstream regulatory mechanism and signaling pathway of PSMC2 in SKCM cells. A Human apoptosis antibody array was performed to analyze the differential expression of human apoptotic markers in SK-MEL-28 cells between shPSMC2 and shCtrl groups. B The relative protein levels of 43 human apoptotic markers in SK-MEL-28 cells between shPSMC2 and shCtrl groups. FC, fold change. C Gray value analysis for five differential expression proteins (DR6, IGFBP-4, p21, p53, and TRAILR-3) in SK-MEL-28 cells between shPSMC2 and shCtrl groups. D Pathway enrichment gene sets of the Wnt signaling pathway in SKCM were shown in snapshoot of GSEA. E Western blot was used to detect the expression of GSK-3 $\beta$ and $\beta$-catenin in A375 and SK-MEL-28 cells after shPSMC2 transfection. Each independent experiment was repeated at least three times. Data were presented as mean \pm SD from three independent experiments. ${ }^{*} p<0.05,{ }^{* *} p<0.01$ and ${ }^{* * *} p<$ 0.001 . 
antibody array Kit (Abcam, Cambridge, MA) according to the manufacturer's protocols.

\section{Gene set enrichment analysis (GSEA)}

Gene Set Enrichment Analysis (GSEA) (http://software.broadinstitute.org/ gsea) was conducted based on the pathway gene set Kyoto Encyclopedia of Genes and Genomes (KEGG) (https://www.kegg.jp/kegg/), which were used to implement gene set enrichment analysis. The expression data of total normalized mRNAs were uploaded to the GSEA v3.0 software. The default weighted enrichment statistic was adopted to process the data 1000 times, with normalized $P<0.05$ considered to be significantly enriched. Next, the highest upregulated results of the GSEA reports were selected to undergo graphics processing using the "ggplot2" package in $\mathrm{R}$.

\section{RNA extraction and RT-qPCR}

For total RNA extraction, tissues and cultured cells were directly lysed using TRIzol reagent (Thermo Fisher Scientific) after medium removal. The extracted RNA was reverse-transcribed into CDNA using a PrimeScript ${ }^{\text {TM }}$ RT Reagent Kit (TaKaRa), with the manufacturer's instructions. The obtained CDNA samples were diluted and used for real-time quantitative PCR (RTqPCR) with SYBR ${ }^{\circ}$ Premix Ex Taq $^{T M}$ II (TaKaRa) (Thermo Fisher Scientific). GAPDH was measured as the internal control. The relative expression level of genes was calculated using the $2^{-\triangle \triangle \mathrm{ct}}$ method.

\section{Protein extraction and Western blot analysis}

RIPA lysis buffer supplemented with protease inhibitor (Roche) and phosphatase inhibitor (Roche) was directly added to cell layers and extracted total proteins. Protein concentrations were determined using the BCA protein assay kit (Beyotime). Equal volume and quantity of protein samples were electrophoresed on 10\% SDS-PAGE and then transferred onto polyvinylidene difluoride (PVDF) membranes (Millipore). The membranes were blocked with $5 \%$ skimmed milk in TBST at room temperature for one $\mathrm{h}$ and incubated with appropriate primary antibodies at $4{ }^{\circ} \mathrm{C}$ overnight. The next day, the membranes were washed with TBST three times and incubated with appropriate secondary HRP antibodies at room temperature for one $\mathrm{h}$. The membrane was rewashed with TBST three times, followed by incubation with appropriated HRP-conjugated secondary antibody. The subsequent immunoblot analysis was performed using enhanced chemiluminescence (ECL). The protein levels were normalized to $\mathrm{GAPDH}$, and the densitometry was measured using Image J software.

\section{Statistical analysis}

The data are expressed as the mean \pm standard deviation (SD) and analyzed by GraphPad Prism 8.0 software. The statistical significance of the data between groups was compared using Student's $t$-test or one-way ANOVA. Relationship between PSMC2 expression and clinical characteristics in patients with malignant melanoma using Mann-Whitney $U$ analysis. $P<0.05$ was defined as statistically significant.

\section{DATA AVAILABILITY}

The datasets used and/or analyzed during the current study are available from the corresponding author on reasonable request.

\section{REFERENCES}

1. Coricovac D, Dehelean C, Moaca E-A, Pinzaru I, Bratu T, Navolan D, et al. Cutaneous Melanoma-A Long Road from Experimental Models to Clinical Outcome: A Review. International Journal of Molecular Sciences. 2018;19:1566.

2. Atkinson V. Recent advances in malignant melanoma. Intern Med J. 2017:47:1114-21.

3. Zhang $M$, Zhang N. Clinical and prognostic factors in 98 patients with malignant melanoma in China. J Int Med Res. 2017;45:1369-77.

4. Knackstedt T, Knackstedt RW, Couto R, Gastman B. Malignant melanoma: diagnostic and management update. plastic and reconstructive surgery. 2018;142:202e-216e.

5. Valko-Rokytovska M, Bruchata K, Simkova J, Milkovicova M, Kostecka Z. Current trends in the treatment of malignant melanoma. Neoplasma 2016;63:333-41.

6. Ribas A, Hamid O, Daud A, Hodi FS, Wolchok JD, Kefford R, et al. Association of pembrolizumab with tumor response and survival among patients with advanced melanoma. Jama. 2016;315:1600-9.
7. Nijhawan D, Zack Travis I, Ren Y, Strickland Matthew R, Lamothe R, Schumacher Steven $E$, et al. Cancer vulnerabilities unveiled by genomic loss. Cell 2012;150:842-54.

8. He J, Xing J, Yang X, Zhang C, Zhang Y, Wang H, et al. Silencing of proteasome $26 \mathrm{~S}$ subunit ATPase 2 regulates colorectal cancer cell proliferation, apoptosis, and migration. Chemotherapy 2019;64:146-54.

9. Song M, Wang $\mathrm{Y}$, Zhang Z, Wang S PSMC2 is up-regulated in osteosarcoma and regulates osteosarcoma cell proliferation, apoptosis and migration. Oncotarget; 8 , No 1. 2016.

10. Qin J, Wang W, An F, Huang W, Ding J. PSMC2 is up-regulated in pancreatic cancer and promotes cancer cell proliferation and inhibits apoptosis. J. Cancer. 2019;10:4939-46.

11. Cui F, Wang Y, Wang J, Wei K, Hu J, Liu F, et al. The up-regulation of proteasome subunits and lysosomal proteases in hepatocellular carcinomas of the HBx gene knockin transgenic mice. Proteomics 2006;6:498-504.

12. Wang $X$, Dong C, Sun L, Zhu L, Sun C, Ma R, et al. Quantitative proteomic analysis of age-related subventricular zone proteins associated with neurodegenerative disease. Sci. Rep. 2016;6:37443.

13. Evan $\mathrm{Gl}$, Vousden $\mathrm{KH}$. Proliferation, cell cycle and apoptosis in cancer. Nature 2001;411:342-8.

14. Khan MM, Xiao J, Patel D, LeDoux MS. DNA damage and neurodegenerative phenotypes in aged Ciz1 null mice. Neurobiol. aging. 2018;62:180-90.

15. Bukholm IK, Nesland JM. Protein expression of p53, p21 (WAF1/CIP1), bcl-2, Bax, cyclin D1 and pRb in human colon carcinomas. Virchows Arch. 2000;436:224-8.

16. North S, El-Ghissassi F, Pluquet O, Verhaegh G, Hainaut P. The cytoprotective aminothiol WR1065 activates p21waf-1 and down regulates cell cycle progression through a p53-dependent pathway. Oncogene 2000;19:1206-14.

17. Dai M, Al-Odaini AA, Fils-Aimé N, Villatoro MA, Guo J, Arakelian A, et al. Cyclin D1 cooperates with p21 to regulate TGF $\beta$-mediated breast cancer cell migration and tumor local invasion. Breast Cancer Res. 2013;15:3246.

18. Qian X, Hulit J, Suyama K, Eugenin EA, Belbin TJ, Loudig O, et al. p21CIP1 mediates reciprocal switching between proliferation and invasion during metastasis. Oncogene 2013;32:2292-303.

19. Bunz F, Dutriaux A, Lengauer C, Waldman T, Zhou S, Brown JP, et al. Requirement for p53 and p21 to sustain G2 arrest after DNA damage. Science 1998;282:1497-501.

20. Xu D, Liu X, Wang Y, Zhou K, Wu J, Chen JC, et al. Identification of immune subtypes and prognosis of hepatocellular carcinoma based on immune checkpoint gene expression profile. Biomedicine Pharmacother. = Biomedecine pharmacotherapie. 2020;126:109903.

21. Koyano T, Namba M, Kobayashi T, Nakakuni K, Nakano D, Fukushima M, et al. The p21 dependent G2 arrest of the cell cycle in epithelial tubular cells links to the early stage of renal fibrosis. Sci. Rep. 2019;9:12059.

22. Kumar S. P53 induction accompanying G2/M arrest upon knockdown of tumor suppressor HIC1 in U87MG glioma cells. Mol. Cell. Biochem. 2014;395:281-90.

23. Levine AJ. p53, the Cellular Gatekeeper for Growth and Division. Cell 1997;88:323-31.

24. Wang Q, Pan F, Li S, Huang R, Wang X, Wang S, et al. The prognostic value of the proteasome activator subunit gene family in skin cutaneous melanoma. J. Cancer. 2019;10:2205-19.

25. Nusse $\mathrm{R}$, Clevers $\mathrm{H}$. Wnt/ $\beta$-catenin signaling, disease, and emerging therapeutic modalities. Cell 2017;169:985-99.

26. Luan W, Wang Y, Chen X, Shi Y, Wang J, Zhang J, et al. PKM2 promotes glucose metabolism and cell growth in gliomas through a mechanism involving a let-7a/ c-Myc/hnRNPA1 feedback loop. Oncotarget;6:13006-18.

\section{ACKNOWLEDGEMENTS}

Not applicable. This study was funded by the National Key R\&D Program of China (2016YFC1100300).

\section{AUTHOR CONTRIBUTIONS}

YW.Y. and FZ.Q. designed this work and wrote the main manuscript text. YW.Y., CY.W., JQ. L., and Y.Z. performed the experiments. YW.Y., WJ.L., and JY.G. prepared figures. All authors reviewed the paper.

\section{ETHICAL APPROVAL}

This study was approved by the Ethics committee of the Zhongshan Hospital Biomedical Research Department. Written informed consents were signed and obtained from all participants, and the study conformed to the ethical principles set forth by the Declaration of Helsinki. 


\section{COMPETING INTERESTS}

The authors declare no competing interests.

\section{ADDITIONAL INFORMATION}

Supplementary information The online version contains supplementary material available at https://doi.org/10.1038/s41420-021-00727-2.

Correspondence and requests for materials should be addressed to Fazhi Qi.

Reprints and permission information is available at http://www.nature.com/ reprints

Publisher's note Springer Nature remains neutral with regard to jurisdictional claims in published maps and institutional affiliations.
Open Access This article is licensed under a Creative Commons Attribution 4.0 International License, which permits use, sharing, adaptation, distribution and reproduction in any medium or format, as long as you give appropriate credit to the original author(s) and the source, provide a link to the Creative Commons license, and indicate if changes were made. The images or other third party material in this article are included in the article's Creative Commons license, unless indicated otherwise in a credit line to the material. If material is not included in the article's Creative Commons license and your intended use is not permitted by statutory regulation or exceeds the permitted use, you will need to obtain permission directly from the copyright holder. To view a copy of this license, visit http://creativecommons. org/licenses/by/4.0/.

C The Author(s) 2021 